abnormal high-level duodenal compartment. When duodenum coarses between the abdominal aorta and superior mesenteric artery (duodenal interposition), the left renal vein entrapment occurs even at $<90$ aortomesenteric degrees.

Three-dimensional PEEK printed extravascular stent was chosen to elevate the superior mesenteric artery and lower the duodenum position, thus relieving left renal vein compression. This extravascular application has significant advantages over open surgery, endovascular stenting and artificial vessel procedures with expanded polytetrafluoroethylene. It provides better cellular vitality by ensuring soft tissue proliferation. By reducing external acceleration and centrifugal force, a three-dimensional printed PEEK extravascular stent reduces adverse side effects. Such a stent has a distinctive personalized design, good stiffness, and durability that allows blood vessel growth, preventing stent migration and thrombosis. Therefore, it is suitable for both adult and pediatric patients. According to the abdominal ultrasound and MSCT scan, the post-operative follow-up results are satisfactory one year after surgery. The patient feels well, the blood flow in the left renal vein is not obstructed, and the blood flow velocity was average. The external stent is in place.

\section{COMPARING POINT OF CARE URINALYSIS TO LABORATORY URINALYSIS AT MATER DEI HOSPITAL}

Warren Debono*, Melise Mifsud, Amaris Spiteri. MDH

\subsection{6/archdischild-2021-europaediatrics.370}

A Study was done to analyse the reliability of point of care urinalysis done at Mater Dei Hospital Paediatric Emergency Department, which is the main hospital in Malta.

Data was collected over a 6 week period, starting from the 18th of January 2021. Reliability was assessed through comparison of the result from the point of care test to the result from the laboratory.

Furthermore if there was evidence of a Urinary Tract Infection (UTI) a urine culture and sensitivity test was performed.

Data was collected by going through the documentation of all the patients that attended the Paediatric Emergency Department during that time period.

If urine was collected for urinalysis and tested by both point of care urinalysis and official laboratory urinalysis, this was included in the data. In the presence of evidence of a UTI data regarding the culture and sensitivity was recorded. The data obtained from the laboratory was then converted to the same units as those obtained from the urinalysis done at the Paediatric Emergency Department. These were then compared and a Chi squared test was performed obtaining a $\mathrm{P}$ value for each of the subset of data collected.

Null Hypothesis - 'There is no significant difference in the values obtained from a urinalysis done at the Paediatric Emergency department and that done at the Laboratory'

$\mathrm{P}$ values obtained for each category were as follows:

Leukocytes - 0.757 Nitrites - 1 Proteins - 0.312 Erythrocytes -0.766 Ketones -0 Glucose -1

The Null hypothesis could be accepted for Leukocytes, Nitrites, Proteins, Erythrocytes and Glucose and rejected for ketones with a confidence of $\mathrm{p}=0.05$

A point of care urinalysis performed at the Paediatric emergency department is reliable to determine the presence and give a measure respectively of WCC, erythrocytes, protein and glucose. It is also reliable to detect nitrites.

The identification and quantification of ketones from a urinalysis at the Paediatric Emergency department should be backed up by a formal urinalysis performed by the laboratory.

\section{ARE THESE REALLY STONES?}

Susana Correia de Oliveira*, Francisca Dias de Freitas, Patrícia Sousa, António Maurício Fonseca, Ricardo Ramires, Cláudia Tavares. Department of Pediatrics, Hospital Senhora da Oliveira - Guimarães, Portugal

\subsection{6/archdischild-2021-europaediatrics.371}

Introduction Nephrolithiasis' recurrence is frequent and increases if an underlying metabolic abnormality is present. Although in up to $15-25 \%$ of affected children there is no risk factor identified, suspicion should be raised in the presence of exaggerated clinical manifestations with no reasonable explanation.

Case Report A eleven-year old uncircumcised adolescent male, with a family history of IgA Nephropathy, was referred to a pediatric nephrology consultation after being seen multiple times in the emergency department with chief complaint of recurrent episodes of dysuria with emission of up to seventeen $3-4 \mathrm{~mm}$ black calculus. He had no lumbar or abdominal pain, nor gross hematuria. On physical examination, Murphy's sign was negative bilaterally and rectal examination was normal. His laboratory evaluation, urinalysis and ultrasound were unremarkable and did not reveal any morphological or functional alterations. A 24-hour urine collection was performed with an elevated concentration of urinary urea but no other noteworthy alterations.

Homocysteine, methionine and homogentisic acid dosing were within normal ranges. Multiple laboratory evaluations of the stones weren't able to determine its composition. No metabolic or infectious cause for a calculus was identified.

Due to the recurrence of this situation he repeated both 24-hour urine collection and ultrasound with no significant results. The calculus was eventually sent to an outside laboratory and rubber elastomer was detected.

Careful examination of the patient's past medical history revealed he had already been evaluated in a Pedopsychiatric consultation before. After determining the stones were selfinduced, the adolescent was once more referred to Pedopsychiatry appointment. No further signs or symptoms of nephrolithiasis were reported.

Discussion Although Factitious Diseases (FD) caused by proxy have been increasingly recognized, there is a significant less awareness that children and adolescents can intentionally falsify symptoms. Even though urinary tract involvement is rare, any unexplained medical condition warrants the exclusion of self-induced FD.

\section{EVOLUTION OF EPIDEMIOLOGICAL AND BACTERIOLOGICAL PROFILES OF FEBRILE URINARY TRACT INFECTIONS IN CHILDREN}

Rania Ben Rabeh*, Sofiène Atiattalah, Salem Yahyaoui, Nada Missaoui, Msaddek Assidi, Sonia Mazigh, Samir Boukthir. Béchir Hamza Children's Hospital

10.1136/archdischild-2021-europaediatrics.372 
Introduction Febrile urinary tract infections (FUTIs) are a common reason of consultation and hospitalization in pediatric clinical practice generating important health care costs. Strains of multidrug-resistant bacteria responsible for FUTIs are currently emerging, which can, in many cases, lead to therapeutic dead-ends. However, the changing epidemiological and bacteriological profiles of FUTIs in children over the past years haven't been studied in Tunisia.

The main purpose of this study was to analyze the evolution of epidemiological and bacteriological profiles of FUTIs in children.

Methods It was a descriptive, analytical and retrospective study over two periods (2000-2005 and 2010-2015), conducted in the department of pediatrics C at the children's hospital Bechir Hamza of Tunis. We included all children who were hospitalized for confirmed FUTIs.

Results This study enrolled 254 patients divided into 2 groups: group 1 which included 105 patients from the first study period and group 2 which included 149 patients of the second period of study. The average age was significantly lower in group $2 \quad(51.2 \pm 39.7$ months versus $32.2 \pm 38.4$ months; $\mathrm{p}<0.001$ ) with a female predominance in both groups. A history of malformativeuropathies and UTIs was more common in the first study group.

Clinical features were similar in both groups. Escherichia coli (E. coli) was the most frequently isolated germ. The antibiograms showed an increase in the resistance rates of E. coli to ampicillin, third generation cephalosporins and gentamicin during the second period of study as compared to the first period. Imipenem and amikacin kept a zero resistance rate between the two periods of the study.

Conclusion Antibiotic resistance rates in upper UTIs have increased significantly over the past years. Preventing those risks requires a more rational prescription of antibiotics.

\section{A SYSTEMATIC REVIEW OF TRANSITIONAL CARE MODELS IN PAEDIATRIC RENAL TRANSPLANT RECIPIENTS}

'Dermot Wildes*, ${ }^{1} \mathrm{CS}$ Costigan, ${ }^{1} \mathrm{~N}$ Dolan, ${ }^{1,2} \mathrm{M}$ Riordan, ${ }^{1} \mathrm{C}$ Sweeney, ${ }^{1} \mathrm{M}$ Stack ${ }^{1} \mathrm{M}$ Waldron, ' $\mathrm{I}$ Flynn, ${ }^{1} \mathrm{M}$ Kinlough, ${ }^{1,2,3} \mathrm{~A}$ Awan. ${ }^{1}$ The Department for Paediatric Nephrology and Transplantation, Children's Health Ireland at Temple St., Temple Street, Dublin 1, Ireland; ' ${ }^{2}$ Department of Paediatrics, Royal College of Surgeons in Ireland, $123 \mathrm{St}$. Stephen's Green, Dublin 2, Ireland; ${ }^{3}$ Department of Paediatrics, UCD School of Medicine, University College Dublin, Belfield, Dublin 4, Ireland

10.1136/archdischild-2021-europaediatrics.373

Adolescence is a time of significant change and challenge for clinicians, parents, and patients. The Paediatrician must ensure patients have the necessary skills and knowledge required to function as an individual, with autonomy and self-care. It is well established that adolescent renal transplant recipients have a high incidence of acute allograft rejection and loss. ${ }^{1}$ The transfer from paediatric to adult care carries an increased risk of graft-related complications attributable to a multitude of reasons, particularly non-adherence to immunosuppressant medicines and poor attendance at scheduled appointments. ${ }^{2-6}$ We aim to present a summary of published, peer-reviewed transitional care models for adolescent renal transplant recipients with a description of their respective approaches.

A systematic review was performed, in a methodology outlined by the PRISMA guidelines. ${ }^{7}$ MEDLINE OVID \&
EMBASE databases were searched for studies that outlined valid, replicable models pertaining to transitional care of paediatric renal transplant recipients between 1946 and Q1 2021. No language restrictions were applied and the reference lists of selected articles were also perused for further eligible studies. Two investigators assessed all studies for eligibility and independently performed data extraction, any discrepancies were settled by consensus.

A total of 1,114 abstracts were identified, which was reduced to 1,020, upon removal of duplicates yielded by the respective searches. We also consulted experts in the field for further eligible articles. A total of 49 articles were deemed appropriate for full-text review and critical appraisal. Eight articles were included in qualitative synthesis. Data were extracted from all included models in an endeavour to compare the approach taken in each respective case as follows: Country; clinical setting; transition facilitators; age at first transition clinic; age at transition.

Despite the well-established need for good transitional care in paediatric solid-organ transplant recipients, models tailored specifically for renal transplant recipients are few in number. Amongst those present, there is a notable degree of heterogeneity in approach. Further research and validation studies of these models are required, to ascertain the most efficacious method of providing effective transitional care to these patients.

\section{REFERENCES}

1. Dobbels F, Ruppar T, De Geest S, Decorte A, Van Damme-Lombaerts R, Fine RN Adherence to the immunosuppressive regimen in pediatric kidney transplant recipients: A systematic review. Pediatr Transplant 2010;14:603-613.

2. Keith DS, Cantarovich M, Paraskevas S, et al. Recipient age and risk of chronic allograft nephropathy in primary deceased donor kidney transplant. Transp/ Int 2006;19:649-656.

3. Dew MA, Dabbs AD, Myaskovsky $L$, et al. Meta-analysis of medical regimen adherence outcomes in pediatric solid organ transplantation. Transplantation 2009;88:736-746.

4. Watson AR. Non-compliance and transfer from pediatric to adult transplant unit Pediatr Nephrol 2000;14:469- 472.

5. Van Arendonk KJ, Boyarsky BJ, Orandi BJ, et al. National trends over 25 years in pediatric kidney transplant outcomes. Pediatrics 2014;133(4):594- 601.

6. Crowley R, Wolfe I, Lock K, McKee M. Improving the transition between paediatric and adult healthcare: A systematic review. Arch Dis Child 2011;96(6): 548553.

7. Moher D, Liberati A, Tetzlaff J, Altman DG, The PRISMA Group. Preferred reporting items for systematic reviews and meta-analyses: the PRISMA statement. PLoS Med 2009;6(7):e1000097. doi:10.1371/journal.pmed1000097

\section{PLASMA EXCHANGE IN PAEDIATRIC NEPHROLOGY - THE IRISH EXPERIENCE}

${ }^{1}$ Dermot Wildes*, ${ }^{1} \mathrm{C}$ Devlin, ${ }^{1} \mathrm{CS}$ Costigan, ${ }^{1} \mathrm{~J} \mathrm{Cox},{ }^{1} \mathrm{C}$ Hensey, ${ }^{1} \mathrm{M}$ Waldron, ${ }^{1} \mathrm{~N}$ Dolan, ${ }^{1} \mathrm{M}$ Riordan, ${ }^{1} \mathrm{C}$ Sweeney, ${ }^{1} \mathrm{M}$ Stack, ${ }^{1} \mathrm{~T}$ Raftery, ${ }^{2} \mathrm{M}$ Cotter, ${ }^{1} \mathrm{~A}$ Awan. ${ }^{1}$ The Department for Paediatric Nephrology and Transplantation, Children's Health Ireland, Temple St., Dublin 1, Ireland; 'The Department of Haematology, Children's Health Ireland at Temple St., Temple Street, Dublin 1, Ireland

\subsection{6/archdischild-2021-europaediatrics.374}

Plasma exchange (PLEX) has proven an effective modality in the management of many paediatric renal conditions. Despite its widespread acceptance, and advancements in the practice of apheresis, there remains a paucity of data pertaining to the paediatric patient population. The use of PLEX in paediatric patient cohorts is technically more challenging than in their adult counterparts, due to numerous factors which include the patient's size, extracorporeal circulatory volume, ability to 